Research Article

\title{
Mitochondrial genetic variability of Didelphis albiventris (Didelphimorphia, Didelphidae) in Brazilian localities
}

\author{
Luciene C.C. Sousa ${ }^{1}$, Célia M.F. Gontijo ${ }^{2}$, Helbert A. Botelho ${ }^{2}$ and Cleusa G. Fonseca ${ }^{1}$ \\ ${ }^{1}$ Departamento de Biologia Geral, Instituto de Ciências Biológicas, \\ Universidade Federal de Minas Gerais, Belo Horizonte, MG, Brazil. \\ ${ }^{2}$ Laboratório de Leishmanioses, Centro de Pesquisas René Rachou, Fundação Oswaldo Cruz, \\ Belo Horizonte, $M G$, Brazil.
}

\begin{abstract}
Didelphis albiventris is a well- known and common marsupial. Due to its high adaptability, this very widespread generalist species occurs under various environmental conditions, this even including protected regions and disturbed urban areas. We studied a $653 \mathrm{bp}$ fragment of cytochrome oxidase c (COI) from 93 biological samples from seven Brazilian localities, with linear distances ranging between 58 and about $1800 \mathrm{~km}$ to analyze the effects of geographic distances on variability and genetic differentiation. The haplotype network presented nine haplotypes and two genetic clusters compatible with the two most distant geographic areas of the states of Minas Gerais, in the southeast, and Rio Grande do Sul, in the extreme south. As each cluster was characterized by low nucleotide and high haplotype diversities, their populations were obviously composed of closely related haplotypes. Surprisingly, moderate to high $\mathrm{F}_{\mathrm{ST}}$ differentiation values and a very weak phylogeographic signal characterizes interpopulation comparisons within Minas Gerais interdemes, these being correlated with the presence of privative haplotypes. On a larger geographic scale, a comparison between demes from Minas Gerais and Rio Grande do Sul presented high $\mathrm{F}_{\mathrm{ST}}$ values and a robust phylogeographic pattern. This unexpected scenario implies that mtDNA gene flow was insufficient to maintain population cohesion, reflected by the observed high differentiation.
\end{abstract}

Key words: Didelphis albiventris, marsupial, variability, COI, genetic differentiation.

Received: November 27, 2011; Accepted: February 28, 2012.

\section{Introduction}

The white-eared opossum Didelphis albiventris Lund, 1840 (Didelphimorphia, Didelphidae) is widely distributed throughout Brazil, Paraguay, Uruguay, Argentina, Bolivia (Gardner, 2008; Costa et al., 2008), Ecuador, Peru and Colombia (Wilson and Reeder, 2005). The species is listed as "Least Concern" in the IUCN Red List Category (Costa et al., 2008). Through their presence in a wide variety of habitats, and adaptability to disturbed areas, such as large towns and other urban habitats, D. albiventris manifests the capacity of coexisting with environmental impacts caused by human exploitation of natural spaces. Another characteristic is their importance as parasite reservoirs, highly relevant in populated urban areas (Schallig et al., 2007).

One of the most important factors affecting mammals in small fragments is the lack of food resources. Little is

Send correspondence to Luciene C.C. Sousa. Departamento de Biologia Geral, Instituto de Ciências Biológicas, Universidade Federal de Minas Gerais, Avenida Antônio Carlos 6627, Pampulha, 31270-901 Belo Horizonte, MG, Brazil. E-mail: lucienecsousa@gmail.com. known on Neotropical forest mammal movement between forest-patches. Even so, anecdotal evidence indicates facile mobility in some species, especially habitat generalists, as opossums (Chiarello, 2000). This was apparent in a southeastern Brazilian Atlantic Forest region, where D. aurita manifested interfragment movement in $19.4 \%$ of recaptures, the highest, when compared to seven other small mammals (Pires et al., 2002). Didelphis are polygynous, the females presenting more stable home ranges and the males migrating more, hence the differences among sexes in the use of space (Loretto and Vieira, 2005), as verified for D. marsupialis in Venezuela, where, on using radiotelemetry methodology, a mean home range 10 times greater for males (122.7 ha) than for females (12 ha) was observed (Sunquist et al., 1987). In this context, white-eared opossums, as seed dispersers, make an important contribution to the maintenance of diverse ecosystems, mainly where specialist frugivores are frequently absent, as in urban forest fragments (Cantor et al., 2010).

In the present survey, four Brazilian biomes in the wide $D$. albiventris distribution were sampled, viz., Atlantic Forest, Cerrado, Caatinga and Pampa. The Atlantic For- 
est and Cerrado appear on the biodiversity hotspots list, which highlights 24 priority conservation areas (Mittermeier et al., 1998). Biodiversity hotspots, occupying only $1.4 \%$ of the Earth's surface, concentrate more than $60 \%$ of terrestrial species (Mesquita, 2004), and mainly consist of heavily exploited and often highly fragmented ecosystems, greatly reduced in extent, and with less than $25 \%$ of the original vegetation remaining (Mittermeier et al., 1998). Several vegetal formations are observed in the Brazilian Atlantic Forest, such as the Seasonal Forest (semi-deciduous and deciduous, the latter occurring on a reduced scale) and the Rain Forest (dense and moist). The seasonal semi-deciduous forest is under extreme risk, formerly caused by sugar cane and coffee plantations, and currently by growing urbanization, especially around the major cities (IBGE, 2011; Fundação SOS Mata Atlântica, 2011). The Araucaria Moist Forest, an endangered ecosystem (only $12.6 \%$ remaining) of the Atlantic Rain Forest, is mostly distributed among small fragments surrounded by anthropogenic habitats, such as cattle pasture, farming and exotic-tree monoculture (Ribeiro et al., 2009; Emer and Fonseca, 2011). The Cerrado, Caatinga and Pampa biomes are characterized by open grassland vegetation. The Cerrado biome, with savanna vegetation, predominates in central Brazil, the Caatinga, with savanna-steppe vegetation, is typical of the semiarid northeast, and the Pampa, restricted to the extreme south, is characterized by steppe vegetation (IBGE, 2011).

Mitochondrial DNA, the most used molecular marker for tracing the geographic distribution of genealogical lineages, even at the intraspecific level, has been consolidated by such characteristics as maternal inheritance and high rates of nucleotide substitution (Avise et al., 1987). Molecular DNA techniques, besides forming the basic tool in population genetics studies for defining taxonomic units (Wilson-Wilde et al., 2010), have also been widely used in mammal diversity surveys when analyzing variability characteristics and distribution, as in the genetic structure analyses of the Atlantic Forest sigmodontine rodents Oligoryzomys nigripes and Euryoryzomys russatus (Gonçalves et al., 2009) and the short-tailed bats Carollia brevicauda, C. perspicillata, $C$. sowelli and C. castanea (Hoffmann et al., 2003). COI, the marker of choice for species discrimination by the Barcode of Life Database (BOLD), is useful for species identification, and the study of differentiation in large-scale structure assaying (Wilson-Wilde et al., 2010; Sousa et al., 2012).

Knowledge on species population genetics is important for a better understanding of species biology, including ecological correlations. The aim here was to study Didelphis albiventris population genetic patterns, by focusing on the geographic distance effect on both variability and genetic differentiation among demes.

\section{Materials and Methods}

\section{Sampling}

This research was developed under a license for scientific purposes granted by IBAMA/SISBIO, number 20170-2, renewed in February, 2011. The institutions that collaborated with sample donations also have their own scientific licenses.

Didelphis albiventris samples from two distant geographic areas in Brazil, herein considered as two geographic clusters, were studied, viz., Minas Gerais (MG), a southeastern state, and Rio Grande do Sul (RS), the southernmost. Linear distances between the studied localities range from 58 to $1795 \mathrm{~km}$ (Table 1). In MG, six localities were sampled (Figure 1). Piracema (Pir) and Almenara (Alm), both in the Atlantic Forest, and Bambuí (Bam), in the Cerrado, were poorly sampled (Figure 1). As the Belo Horizonte Metropolitan Region (BH, 40 samples), Divinópolis (Div, 18 samples) and the Reserva Indígena Xacriabá (RIX, 18 samples) were well-sampled, the hypothesis of separate demes was tested here. Although BH and Div are geographically situated in transitional regions between the Cerrado and Atlantic Forest, both sampled areas present characteristics of the Atlantic Forest biome. RIX, a transitional area connecting the Cerrado and Caatinga biomes, presents ecotone characteristics. The RS geographic cluster (Figure 1) comprised samples collected in two localities in the Araucaria Moist-Rain Forest, a domain of the Atlantic Forest (Machadinho and Caxias do Sul), four collected in the Pampa biome (Porto Alegre and Triunfo) and three from road killed animals from RS, but without exact locality information. For Rio Grande do Sul, distances correspond to the average among known state collection localities (Table 1).

In the entire analysis, and due to the small size of the samples obtained from each location, as a whole, the RS sample group was treated as one single study area.

\section{DNA extraction, amplification and sequencing}

The tissue samples used were mostly obtained from the liver, and in a few cases, the spleen, muscle and blood.

Table 1 - Matrix with linear geographic distances $(\mathrm{km})$ between sampling areas. The meanings of abbreviations are cited in the topic 'sampling' in "Material and Methods".

\begin{tabular}{lccccccc}
\hline Demes & BH & Div & RIX & Bam & Pir & Alm & RS \\
\hline BH & & & & & & \\
Div & 111 & & & & & \\
RIX & 548 & 590 & & & & \\
Bam & 221 & 115 & 600 & & & \\
Pir & 94 & 58 & 621 & 164 & & \\
Alm & 523 & 622 & 393 & 700 & 620 & \\
RS & 1256 & 1180 & 1725 & 1130 & 1170 & 1795 \\
\hline
\end{tabular}




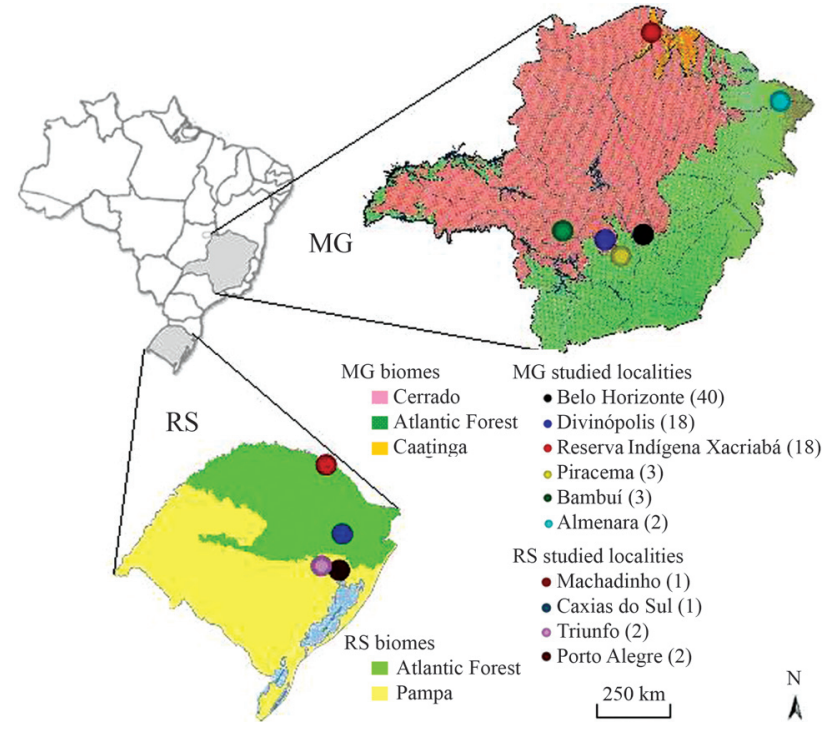

Figure 1 - Minas Gerais, a southeastern state (Drummond et al., 2005), and Rio Grande do Sul, the southernmost state of Brazil (SCP/DEPLAN, 2007), with approximate collection locations, sample numbers (in parentheses) and biome correspondence. Three samples from road killed animals from RS, but without exact information on locality, were not represented.

Ear-tissue fragments were collected from road killed animals. Tissue samples were preserved in $95 \%$ ethanol and stored at $-20^{\circ} \mathrm{C}$. Most of the samples were kindly donated by researchers from the Centro de Pesquisa René Rachou/FIOCRUZ, Museu de Ciências Naturais da Pontifícia Universidade Católica de Minas Gerais, Fundação Zoo-Botânica do Rio Grande do Sul, and the Pontifícia Universidade Católica do Rio Grande do Sul. DNA from macerated tissue fragments was extracted according to standard phenol-chloroform protocols, as described by Sambrook et al. (2001).

DNA sequences of the mitochondrial cytochrome oxidase I gene (COI) were amplified using the universal primers LCO 1490: 5' GGT CAA CAA ATC ATA AAG ATA TTG G 3' and HCO 2198: 5' TAA ACT TCA GGG TGA CCA AAA AAT CA 3' (Folmer et al., 1994). Each PCR was carried out in a $20 \mu \mathrm{L}$ final volume, containing 50 ng of genomic DNA, 10x Buffer III B (Phoneutria: $100 \mathrm{mM}\left(\mathrm{NH}_{4}\right)_{2} \mathrm{SO}_{4}, 100 \mathrm{mM} \mathrm{KCl}, 100 \mathrm{mM}$ Tris- $\mathrm{HCl} \mathrm{pH}$ $8,4,1 \%$ Triton-X, $\left.15 \mathrm{mM} \mathrm{MgCl}_{2} 10 \mathrm{x}\right), 0.8 \mu \mathrm{M}$ of dNTPs, $0.5 \mu \mathrm{M}$ of each primer, $1 \%$ bovine serum albumin (BSA), and 1 unit of Taq DNA polymerase (Phoneutria). After an initial denaturing step of $3 \mathrm{~min}$ at $94{ }^{\circ} \mathrm{C}$, the PCR conditions followed a standard three-step protocol, with 30 cycles of 1 min at $94{ }^{\circ} \mathrm{C}, 45 \mathrm{~s}$ at $47^{\circ} \mathrm{C}$ and $30 \mathrm{~s}$ at $72^{\circ} \mathrm{C}$, followed by a final extension step for $5 \mathrm{~min}$ at $72{ }^{\circ} \mathrm{C}$. Satisfactory amplifications were visualized in $6 \%$ polyacrylamide gels. Amplified DNA products were purified using $20 \%$ polyethylene-glycol (PEG 8000 ) and $2.5 \mathrm{M} \mathrm{NaCl}$, according to Sambrook et al. (2001).

PCR products were sequenced in both directions with the same primers, LCO 1490 or HCO 2198 (Folmer et al.,
1994) on an ABI3100 automated sequencer using a BigDye Terminator Kit v3 (Applied Biosystems). Alternatively, some sequences were obtained on a MegaBACE automated capillary sequencer, using an ET dye terminator kit (GE Healthcare).

\section{Statistical data analysis}

Sequences were base-called with Phred software (Ewing et al., 1998; Ewing and Green, 1998), and checked for quality with Phrap software (Green, 1994), whereas the assembled chromatograms were checked and edited in Consed (Gordon et al., 1998). Chromatogram peaks for each sequence were visually verified to ensure consensus fidelity. Sequence groups were aligned using the Clustal W algorithm implemented in MEGA 4.1 (Tamura et al., 2007 ), with a $653 \mathrm{bp}$ fragment showing high levels of sequence quality for all individuals. The studied sequences were deposited in GenBank (accession numbers JN638891 to JN 638983).

MEGA 4.1 (Tamura et al., 2007), DNAsp v. 5 (Librado and Rozas, 2009) and Arlequin v. 3.1 (Excoffier et $a l ., 2005)$ were used for analyzing intrapopulation genetic diversity and estimating standard indices of genetic variation, such as haplotype $(\mathrm{Hd})$ and nucleotide $(\pi)$ diversities. Arlequin v. 3.1 (Excoffier et al., 2005) was also used for calculating differentiation indices and analyzing molecular variance (AMOVA), with the Tamura \& Nei distance method and 10,100 permutations. This software was also used for calculating Mismatch Distribution, Tajima's D and Fu's Fs tests of neutrality, thereby assaying demographic expansion, and whether mutations were neutral or under the influence of selection.

The haplotype network was constructed based on statistical parsimony. The maximum number of steps parsimoniously connecting two haplotypes was informed by TCS v. 1.21, which estimates genealogical relationships among sequences (Clement et al., 2000).

Alleles in Space (AIS) (Miller, 2005) was used for analyzing the relationship between inter-individual spatial and genetic information, and Mantel testing and spatial autocorrelation analysis for predicting patterns, such as correlations between genetic and geographical distances.

The best evolutionary model was determined with Modeltest v. 3.7 (Posada and Crandall, 1998). Phylogeographic inference using Maximum Parsimony, Maximum Likelihood and Bayesian analyses were carried out with PAUP* 4.0 (Swofford, 2002), PHY ML 3.0 (Guindon and Gascuel, 2003) and MrBayes (Huelsenbeck and Ronquist, 2001), respectively.

\section{Results}

\section{Molecular characterization of mtDNA COI fragments}

The analysis of $93 \mathrm{mtDNA}$ COI sequences revealed 24 polymorphic sites, all of which corresponding to synon- 
ymous transitions. Nucleotide composition was 34.1\% thymine, $22.4 \%$ cytosine, $28.2 \%$ adenine and $15.3 \%$ guanine. Nine haplotypes $(\mathrm{H}=9)$, all with three or more recordings of occurrence, were observed. Haplotype 1 occurred throughout all the areas studied in $\mathrm{MG}$, whereas seven were private to just one analyzed area, viz., haplotypes 2 and 3 to Belo Horizonte (BH), 4 and 5 to Reserva Indígena Xacriabá (RIX), 7 to Divinópolis (Div), and 9 and 10 to Rio Grande do Sul State (RS) (Table 2).

Haplotype diversity (Hd) of 0.7235 (with a standard deviation of 0.044 ) and nucleotide diversity $(\pi)$ of 0.0065 , characterized the analyzed data set (Table 3 ). Hd and $\pi$ for each population can be seen in Table 3 .

When RS sequences were excluded, and only the six MG localities analyzed, nine polymorphic sites were found, these corresponding to seven haplotypes $(\mathrm{H}=7)$, haplotype diversity $(\mathrm{Hd})$ of $66.52 \%$, and nucleotide diversity $(\pi)$ of $0.23 \%$.

\section{Genetic differentiation among populations}

The haplotype network produced with the $93 \mathrm{D}$. albiventris specimens using statistical parsimony, and with a $91 \%$ connection limit, showed two distant genetic clusters compatible with the MG and RS geographic clusters (Figure 2).

Highly significant population pairwise differentiation values were observed (Table 4). On comparing demes between geographic clusters (RS x MG), obtained values proved to be higher than $91 \%$, the smallest $\mathrm{F}_{\mathrm{ST}}$ being observed in the comparison RIX x RS (the meanings of abbreviations are cited in "Materials and Methods").

AMOVA indicated genetic structuring $\left(\mathrm{F}_{\mathrm{ST}}=93.6 \%\right.$, $\mathrm{p}=0.000)$. According to the Tamura \& Nei distance method, and on comparing the two geographic clusters (MG and RS), intergroup differences contributed with $91.3 \%$ of the total genetic variance (Table 5), whereas interpopulation variance, within the groups was $2.26 \%$, and within the populations themselves, $6.44 \%$. All the results were significant.

By way of analysis using the Tamura \& Nei distance method, and with $\mathrm{BH}$ and Divinópolis as a first group and
Table 3 - Intrapopulation and total diversities. Number of samples $(\mathrm{N})$, number of haplotypes $(\mathrm{H})$, number of polymorphic sites $(\mathrm{S})$, nucleotide diversity $(\pi)$, haplotype diversity (Hd). The meanings of locality abbreviations are cited in the topic 'sampling' in "Material and Methods".

\begin{tabular}{lccccc}
\hline Locality & $\mathrm{N}$ & $\mathrm{H}$ & $\mathrm{S}$ & $\% \pi$ & $\% \mathrm{Hd}$ \\
\hline BH & 40 & 3 & 3 & 0.17 & 59.10 \\
Divinópolis & 18 & 3 & 3 & 0.11 & 46.41 \\
RIX & 18 & 3 & 4 & 0.3 & 69.94 \\
Bambuí & 3 & 2 & 2 & 0.20 & 66.67 \\
Piracema & 3 & 2 & 2 & 0.20 & 66.67 \\
Almenara & 2 & 1 & 0 & 0 & 0 \\
RS & 9 & 2 & 1 & 0.09 & 55.56 \\
Total & 93 & 9 & 24 & 0.65 & 72.35 \\
\hline
\end{tabular}

RIX as a second, variation partitioning revealed $17.04 \%$ of intergroup variance, $13.21 \%$ of interpopulation within groups, and $69.74 \%$ of intrapopulation, with $\mathrm{F}_{\mathrm{ST}}=30.26 \%$ $(\mathrm{p}=0.000)$ (Table 6).

Two groups were formed, the first comprising $\mathrm{BH}$ and Divinópolis samples and the second RIX (the meanings of abbreviations are cited in "Material and Methods").

Tajima's D $(p>0.35)$ and Fu's $F_{S}$ statistics $(p>0.51)$ neutrality tests were non-significant. As a test of recent population expansion, applied mismatch distribution analysis indicated non-significant bimodal distribution (Figure 3).

On compiling a complete dataset, Mantel test analysis revealed two geographical clusters corresponding to genetic clusters (Figure 4). Although, on analyzing MG and RS populations, genetic and geographical distances were highly correlated $(\mathrm{r}=0.8901$; $\mathrm{P}$ of a correlation greater than or equal to that observed $=0.001)$, they were considerably less so $(r=0.2216$; $P$ of a correlation greater than or equal to that observed $=0.002$ ), when analyzing only MG.

The HKY 85 evolutionary model of nucleotide substitution, together with the Akaike informative criterion in Modeltest 3.7 (Posada and Crandall, 1998), was found to be the most appropriate for dataset analysis. As a whole, phylogeographic analysis with Maximum Parsimony, Maximum Likelihood and Bayesian analysis revealed a weak

Table 2 - Haplotype (Hap) occurrence in populations. The meanings of abbreviations are cited in the topic 'sampling' in "Material and Methods".

\begin{tabular}{lcccccccccc}
\hline Locality & Hap1 & Hap2 & Hap3 & Hap4 & Hap5 & Hap6 & Hap7 & Hap8 & Hap9 & Total \\
\hline BH & 22 & 13 & 5 & 0 & 0 & 0 & 0 & 0 & 0 & 40 \\
Divinópolis & 13 & 0 & 0 & 0 & 0 & 2 & 3 & 0 & 0 & 18 \\
RIX & 6 & 0 & 0 & 7 & 5 & 0 & 0 & 0 & 0 & 18 \\
Bambuí & 1 & 0 & 0 & 0 & 0 & 2 & 0 & 0 & 0 & 3 \\
Piracema & 2 & 0 & 0 & 0 & 0 & 1 & 0 & 0 & 0 & 3 \\
Almenara & 2 & 0 & 0 & 0 & 0 & 0 & 0 & 0 & 0 & 2 \\
RS & 0 & 0 & 0 & 0 & 0 & 0 & 0 & 4 & 5 & 9 \\
Total & 46 & 13 & 5 & 7 & 5 & 5 & 3 & 4 & 5 & 93 \\
\hline
\end{tabular}


phylogeographic pattern for $D$. albiventris, except for $\mathrm{MG}$ and RS, where there was a clear differentiation into two distinct haplogroups (data not shown).

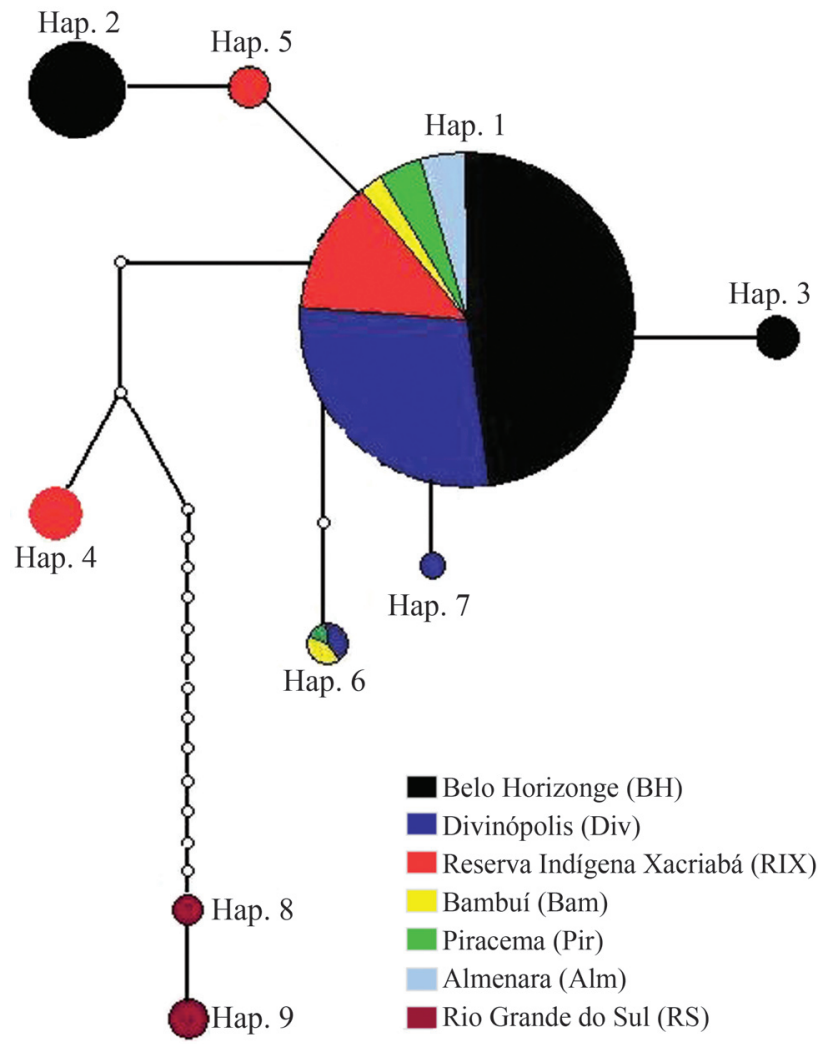

Figure 2 - Haplotype network for D. albiventris using statistical parsimony. Numbered circles represent haplotypes (Hap.), with the circle size corresponding to haplotype frequency. Colors represent the sampling sites. Small open circles indicate missing haplotypes.

Table 4 - Population pairwise $\mathrm{F}_{\mathrm{ST}}$ calculated using the Tamura \& Nei distance method. The meanings of abbreviations are cited in "Material and Methods".

\begin{tabular}{lcc}
\hline Comparison & $\mathrm{F}_{\mathrm{ST}}$ & $\mathrm{p}$ values \\
\hline BH x RIX & 0.2774 & $0.0000 \pm 0.0000$ \\
BH x Div & 0.1935 & $0.0029 \pm 0.0016$ \\
RIX x Div & 0.2790 & $0.0000 \pm 0.0000$ \\
BH x RS & 0.9431 & $0.0000 \pm 0.0000$ \\
Div x RS & 0.9622 & $0.0000 \pm 0.0000$ \\
RIX x RS & 0.9127 & $0.0000 \pm 0.0000$ \\
\hline
\end{tabular}

Table 5 - AMOVA using the Tamura \& Nei distance method, considering MG and RS as groups, and collection localities as populations.

\begin{tabular}{lc}
\hline Source of variation & Percentage of variation \\
\hline Among groups & 91.30 \\
Among populations within groups & 2.26 \\
Within populations & 6.44 \\
\hline
\end{tabular}

$\mathrm{FST}=0.9356 ; \mathrm{p}=0.00 \pm 0.00$.
Table 6 - AMOVA using the Tamura \& Nei distance method, considering $\mathrm{BH}, \mathrm{Div}$ and RIX as populations. The meanings of abbreviations' are cited in "Material and Methods".

\begin{tabular}{lc}
\hline Source of variation & Percentage of variation \\
\hline Among groups & 17.04 \\
Among populations within groups & 13.21 \\
Within populations & 69.74 \\
\hline
\end{tabular}

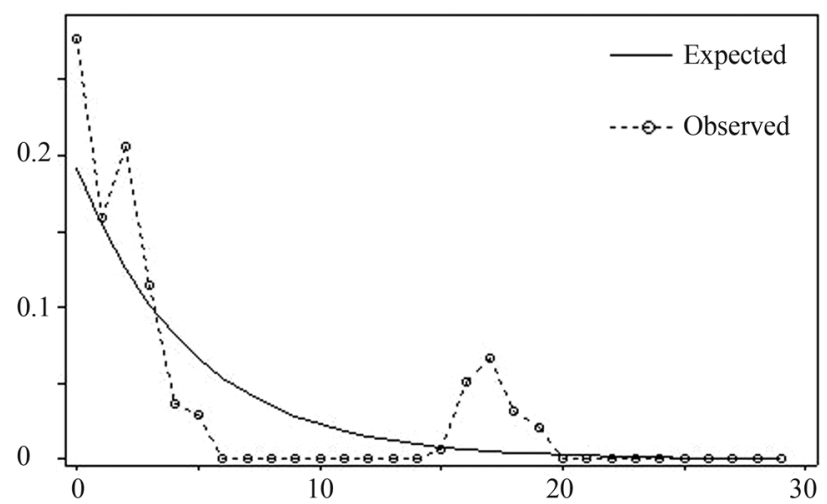

Figure 3 - Mismatch distribution analysis showing bimodal distribution (non-significant).

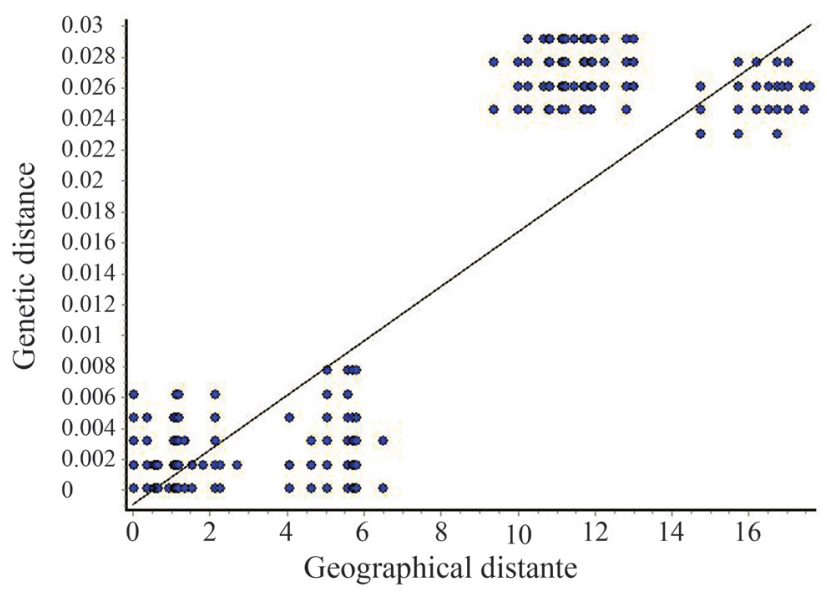

Figure 4 - Mantel Test Results showing correlations between genetic and geographical distances.

\section{Discussion}

As expected when working with a conserved functional gene, all the 24 polymorphic sites in the COI fragment analyzed were synonymous transitions. This situation influences diversity indices, which tend to be considerably lower than for non-coding regions.

Four biomes and several natural conditions were sampled in this survey. In the case of the Atlantic Forest, this involved various threatened ecosystems. In the haplotype network (Figure 2), a large number of steps were observed 
between the haplotypes of the RS Araucaria Atlantic Rain Forest and those of the MG Seasonal Semi-Deciduous Forest, which illustrates the great mtDNA genetic distance between $D$. albiventris haplotypes from both of these ecosystems. As similarly great distances were also observed in all the other biome pairwise comparisons that involved locations from different geographic clusters (MG x RS), an association between geographic and genetic distance is strongly implied. The small genetic distances observed between haplotypes from distinct biomes within each geographic cluster reinforce this argument. As an example, the Rio Grande do Sul haplotypes are cited: the forms from the Araucaria Rain Forest are the same as those occurring in the Pampa biome.

The number of polymorphic sites was considerably greater in the analysis involving all the studied areas (24 variable sites) than in that excluding RS state (only nine). Hence, nucleotide diversity $(\pi)$ was nearly three times greater in the first situation. On analyzing only MG-state samples, the low nucleotide diversity $(\pi)(0.227 \%)$ and haplotype diversity of $66.52 \%$, indicate the presence of haplotypes with few nucleotide differences, thus coherent with the observed haplotype network (Figure 2).

On comparing intrapopulation diversity indices $(\pi$, $H d$ ), it was observed that the highest values were attributed to Reserva Indígena Xacriabá (RIX) in MG state. In accordance, the smallest pairwise $\mathrm{F}_{\mathrm{ST}}$ value obtained between geographic clusters (MG and RS) was when comparing RIX x RS (Table 4). Although unexpected, when considering the effect of distance, this is understandable, when thinking of the higher diversity indices exhibited for this ecotone area, located in a transitional area between the Cerrado and Caatinga biomes. Ecotones may be a source of evolutionary novelty (Smith et al., 1997), playing an important role in the maintenance of genetic diversity, in divergence, and in the speciation process (Kark et al., 2002). Greater attention and higher priority in conservation research and planning should be dedicated to transitional zones which potentially serve as within-species diversity hotspots (Smith et al., 1997; Kark et al., 2002).

By applying statistical parsimony to the haplotype network for 93 D. albiventris specimens two separate genetic clusters, clearly compatible with the two major geographic clusters (MG and RS), could be discerned. The connection between both required a large number of steps, and could only be observed by reducing the connection limit to $91 \%$, this corresponding to a minimum nucleotide distance of 15 steps between haplotypes from the two areas. The observed genetic differentiation was probably the result of distance effect, and is consistent with low mtDNA gene flow, insufficient for maintaining population unity. Haplotypes were genetically close to each other within each geographic cluster.

The $\mathrm{F}_{\mathrm{ST}}$ values between MG and RS genetic clusters, in all the population pairwise comparisons, were significant $(\mathrm{p}=0.00 \pm 0.00)$ and extremely high (FST $>91 \%)$, clearly reflecting population structure, with $91.3 \%$ of intergroup contribution. On a more restricted scale, when comparing demes in the MG geographic cluster alone, a considerable part of variation (69.74\%) was intrapopulation (Table 6), with $\mathrm{F}_{\text {ST }}$ lower than $28 \%(\mathrm{p} \leq 0.0029 \pm 0.0016)$.

According to Edelaar and Björklund (2011), considering $\mathrm{F}_{\mathrm{ST}}$ as a measurement of population differentiation is a misunderstanding, as it actually measures the fixation of alleles. In fact, the observed $\mathrm{F}_{\mathrm{ST}}$ values were surprisingly high, and seemed to much more reflect the presence of deme privative haplotypes than molecular distances between haplotypes.

On comparing only MG zones, phylogeographic analysis revealed a very weak phylogeographic pattern, with a complete shuffling of samples of different origins in all the constructed phylogenies, whereby a pattern with haplotype admixture between localities. The resultant tree showed no separation between localities, even when linearly $700 \mathrm{~km}$ apart. The observed pattern is consistent with those observed in species with a limited or narrow phylogeographic population structure, and life histories conductive to dispersal, occupying ranges without long-term impediments to gene flow (Avise, 2000). Although apparently incompatible with the high to moderate $\mathrm{F}_{\mathrm{ST}}$ values observed, assuredly phylogeographic analysis, although less sensitive to evidence of population differentiation, reflects two important factors, the presence of haplotype 1 in all the MG localities studied, thereby connecting them, and the low number of polymorphic sites separating haplotypes.

In contrast, MG and RS specimen phylogenies confirmed genetic separation between these geographic clusters, thus giving evidence of two spatially circumscribed haplogroups, genetically relatively far apart. This pattern seems to distinguish deep allopatric lineages in a gene tree, probably explained by long-term extrinsic barriers to gene flow (Avise, 2000). Nevertheless, this cannot be interpreted as major phylogeographic discontinuity, since there is a significantly wide sample gap. Thus, this clear separation between specimens from MG and RS in the gene tree, appears to be a result of considerable geographic separation (about $1800 \mathrm{~km}$ of linear distance). If this gap area were studied, a pattern with a weak phylogeographic signal characterizing $D$. albiventris lineage spatial distribution would possibly be found.

Tests of neutrality (the Tajima $\mathrm{D}$ and $\mathrm{Fu} F_{\mathrm{S}}$ statistics) to check excess of rare mutations, as evidence of recent population expansion, were non-significant. Although involved p-values were non-significant, mismatch distribution analysis for testing demographic expansion presented a graph with bimodal distribution, thus consistent with allopatric divergence followed by population growth. This could represent a possible hypothesis for the present study-case. The Mantel test and spatial autocorrelation analysis confirmed the strong correlation ( 0.8901$)$ between 
geographic and genetic distances, when analyzing the $\mathrm{MG}$ and RS clusters. Differentiation probably reflects both the great distance between localities, and the existence of barriers in the wide range of species distribution. As only gene flow can genetically connect populations, the maintenance of COI gene differentiation implies the presence of barriers to mtDNA gene flow, although other important factors seem to be closely related to the observed differentiation results, viz., the bridge between a methodology based on a haplotypic mtDNA system, and ecological characteristics, especially $D$. albiventris sex-biased dispersion.

Due to its wide distribution, generalist habits, high adaptability, capacity to move long distances (Gentile and Cerqueira, 1995; Chiarello, 2000; Pires et al., 2002), and outstanding mobility, when compared to other small mammals (Pires et al., 2002), D. albiventris populations are presumed to be genetically connected. The unexpectedly high differentiation among MG demes seems to be totally unaligned with the above cited ecological characteristics, whence the importance of considering an alternative. A mean home range ten times greater for males (122.7 ha) than for females (12 ha) has been observed for $D$. marsupialis (Sunquist et al., 1987). Although dispersion competence is relevant to promoting genetic approximation between populations, it is in no way a guarantee of gene flow. Even so, as Didelphis dispersion is recognizably greater in males, this probably does indeed contribute more. Working with a maternally inherited genetic marker, it was impossible to discuss complete $D$. albiventris diversity history, since mtDNA analysis told nothing about the male's effective contribution to connecting demes. Hence, our results furnished data only on the mutational history corresponding to maternal lineage genealogical information. The observed mtDNA COI genetic differentiation was consistent with mtDNA gene flow insufficiency in maintaining population unity, or to effectively approximate separated demes in the large geographic scenario studied. Additional research with nuclear markers (microsatellites and/or sequences) could complete our findings, thereby providing a better understanding of species population genetics. As to the female contribution to $D$. albiventris population structure, the haplotype network and differentiation values strongly suggest that female gene flow is insufficient in connecting and effectively approximating the populations under study. The contact with this widespread and important species emphasized the need for additional surveys towards a better understanding of its interesting biology.

\section{Acknowledgments}

We are grateful to the Centro de Pesquisa René Rachou - Fundação Oswaldo Cruz, Célia Gontijo, Helbert Botelho and José Eloy dos Santos Júnior; to the Pontifícia Universidade Católica de Minas Gerais - Museu de Ciências Naturais, Edeltrudes Câmara, Cláudia Costa and Da- nilo Saraiva; to the Fundação Zoo-Botânica do Rio Grande do Sul, Daniela Sanfelice; to the Pontifícia Universidade Católica do Rio Grande do Sul, Eduardo Eizirick and Manoel Rodrigues for specimen donations made for this research. Financial support was provided by the Fundação de Amparo à Pesquisa de Minas Gerais and Programa Ecológico de Longa Duração.

\section{References}

Avise JC, Arnold J, Ball RM, Bermingham E, Lamb T, Neigel JE, Reeb CA and Saunders NC (1987) Intraspecific phylogeography: The mitochondrial DNA bridge between population genetics and systematics. Annu Rev Ecol Syst 18:489-522.

Avise JC (2000) Phylogeography the History and Formation of Species. Harvard University Press, London, $447 \mathrm{pp}$.

Cantor M, Ferreira LA, Silva WR and Setz EZF (2010) Potential seed dispersal by Didelphis albiventris (Marsupialia, Didelphidae) in highly disturbed environment. Biota Neotrop 10:45-51.

Chiarello AG (2000) Density and population size of mammals in remnants of Brazilian Atlantic Forest. Conserv Biol 14:1649-1657.

Clement M, Posada D and Crandall KA (2000) TCS: A computer program to estimate gene genealogies. Mol Ecol 9:16571659.

Drummond GM, Martins CS, Machado ABM, Sebaio FA and Antonini Y (2005) Biodiversidade em Minas Gerais: Um Atlas para sua Conservação. 2nd edition. Fundação Biodiversitas, Belo Horizonte, 222 pp.

Edelaar $\mathrm{P}$ and Björklund $\mathrm{M}$ (2011) If $\mathrm{F}_{\mathrm{ST}}$ does not measure neutral genetic differentiation, then comparing it with QST is misleading. Or is it? Mol Ecol 20:1805-1812.

Emer C and Fonseca CR (2011) Araucaria Forest conservation: Mechanisms providing resistance to invasion by exotic timber trees. Biol Invasions 13:189-202.

Ewing B and Green P (1998) Base-calling of automated sequencer traces using Phred. II. Error probabilities. Genome Res 8:186-194.

Ewing B, Hillier LD, Wendl MC and Green P (1998) Base-calling of automated sequencer traces using Phred. I. Accuracy assessment. Genome Res 8:175-185.

Excoffier L, Laval G and Schneider S (2005) Arlequin ver. 3.0: An integrated software package for population genetics data analysis. Evol Bioinform Online 1:47-50.

Folmer O, Black M, Hoeh W, Lutz R and Vrijenhoek R (1994) DNA primers for the amplification of mitochondrial cytochrome c oxidase subunit I from diverse metazoan invertebrates. Mol Mar Biol Biotech 3:294-299.

Gardner AL (2008) Mammals of South America vol. 1: Marsupials, Xenarthrans, Shrews and Bats. The University of Chicago Press, Chicago, 690 pp.

Gentile R and Cerqueira R (1995) Movement patterns of five species of small mammals in a Brazilian restinga. J Trop Ecol 11:671-677.

Gonçalves GL, Marinho JR and Freitas TRO (2009) Genetic structure of Sigmodontine rodents (Cricetidae) along an altitudinal gradient of the Atlantic Rain Forest in southern Brazil. Genet Mol Biol 32:882-885.

Gordon D, Abajian C and Green P (1998) Consed: A graphical tool for sequence finishing. Genome Res 8:195-202. 
Guindon S and Gascuel O (2003) A simple, fast, and accurate algorithm to estimate large phylogenies by maximum likelihood. Syst Biol 52:696-704.

Hoffmann FG and Baker RJ (2003) Comparative phylogeography of short-tailed bats (Carollia, Phyllostomidae). Mol Ecol 12:3403-3414.

Huelsenbeck JP and Ronquist F (2001) MRBAYES: Bayesian inference of phylogeny. Bioinformatics 17:754-755.

Kark S, Mukerji T, Safriel UN, Noy-Meir I, Nissani R and Darvasi A (2002) Peak morphological diversity in an ecotone unveiled in the chukar partridge by a novel Estimator in a Dependent Sample (EDS). J Anim Ecol 71:1015-1029.

Librado P and Rozas J (2009) DnaSP ver. 5: A software for comprehensive analysis of DNA polymorphism data. Bioinformatics 25:1451-1452.

Loretto D and Vieira MV (2005) The effects of reproductive and climatic seasons on movements in the black-eared opossum (Didelphis aurita Wied-Neuwied, 1826). J Mammal 86:287-293.

Mesquita CAB (2004) RPPN da Mata Atlântica: Um Olhar Sobre as Reservas Particulares dos Corredores de Biodiversidade Central e da Serra do Mar. Conservação Internacional, Belo Horizonte, $48 \mathrm{pp}$.

Miller MP (2005) Alleles in Space Computer software for the joint analysis of inter-individual spatial and genetic information ver. 1.0. J Hered 96:722-724.

Mittermeier RA, Myers N, Thomsen JB, Fonseca GAB and Olivieri S (1998) Biodiversity hotspots and major tropical wilderness areas: Approaches to setting conservation priorities. Conserv Biol 12:516-520.

Pires AS, Lira PK, Fernandez FAS, Schittini GM and Oliveira LC (2002) Frequency of movements of small mammals among Atlantic Coastal Forest fragments in Brazil. Biol Conserv 108:229-237.

Posada D and Crandall A (1998) MODELTEST: Testing the model of DNA substitution. Bioinformatics 14:817-818.

Ribeiro MC, Metzger JP, Martensen AC, Ponzoni F and Hirota M (2009) Brazilian Atlantic forest: How much is left and how is the remaining forest distributed? Implications for conservation. Biol Conserv 142:1141-1153.

Sambrook J, Russel DW and Sambrook J (2001) Molecular Cloning: A Laboratory Manual. CHSL Press, New York.

Schallig HDFH, Silva ES, Van Der Meide WF, Schoone GJ and Gontijo CMF (2007) Didelphis marsupialis (Common Opossum): A potential reservoir host for zoonotic Leishmaniasis in the metropolitan region of Belo Horizonte (Minas Gerais, Brazil). Vector-Borne Zoonotic Dis 7:387-393.

Smith TB, Wayne RK, Girman DJ and Bruford MW (1997) A role for ecotones in generating rainforest biodiversity. Science 276:1855-1857.

Sousa LCC, Gontijo CMF, Lacorte GA, Meireles SN, Silva AP and Fonseca CG (2012) Molecular characterization of an opossum Didelphis albiventris (Marsupialia, Didelphidae) population in an urban fragment of Brazilian Atlantic Rain Forest and support to species barcode identification. Genet Mol Res (in press).

Sunquist ME, Austad N and Sunquist F (1987) Movement patterns and home range in the common opossum (Didelphis marsupialis). J Mammal 68:173-176.

Swofford DL (2002) PAUP*: Phylogenetic analysis using parsimony (and other methods) ver. 4. Sinauer Associates, Sunderland.

Tamura K, Dudley J, Nei M and Kumar S (2007) MEGA4: Molecular evolutionary genetics analysis software ver. 4.0. Mol Biol Evol 24:1596-1599.

Wilson DE and Reeder DAM (2005) Mammal Species of the World: A Taxonomic and Geographic Reference. 3rd edition. The Johns Hopkins University Press, Baltimore, $743 \mathrm{pp}$.

Wilson-Wilde L, Norman J, Robertson J, Sarre S and Georges A (2010) Current issues in species identification for forensic science and the validity of using the cytochrome oxidase I (COI) gene. Forensic Sci Med Pathol 6:233-241.

\section{Internet Resources}

Arlequin Software, http://cmpg.unibe.ch/software/arlequin3/ (July 31, 2011).

DNAsp Software, http://www.ub.edu/dnasp/ (July 31, 2011).

Costa L, Astúa de Moraes D, Brito D, Soriano P, Lew D and Delgado C (2008) Didelphis albiventris. IUCN Red List of Threatened Species 2011.1, http://www.iucnredlist.org/apps/redlist/details/40489/0 (July 31, 2011).

MEGA 4 Software, http://www.megasoftware.net/ (July 31, 2011).

MrBayes Software, http://mrbayes.sourceforge.net/download.php (July 31, 2011).

National Center for Biotechnology Information - GenBank, http://www.ncbi.nlm.nih.gov/genbank/ (July 31, 2011).

Phred, Phrap and Consed Software, http://www.phrap. org/phredphrapconsed.html (July 31, 2011).

Green P (1994) Phrap. Genome Sciences Department, University of Washington, Laboratory of Phil Green, http://www.genome.washington.edu/UWGC/analysistools/phrap.htm (July 31, 2011).

PhyML, http://www.atgc-montpellier.fr/phyml/versions.php (July 31, 2011).

SCP/DEPLAN Secretaria do Planejamento Governo do Rio Grande do Sul, http://www.scp.rs.gov.br (July 31, 2011).

Associate Editor: Louis Bernard Klackzo

License information: This is an open-access article distributed under the terms of the Creative Commons Attribution License, which permits unrestricted use, distribution, and reproduction in any medium, provided the original work is properly cited. 